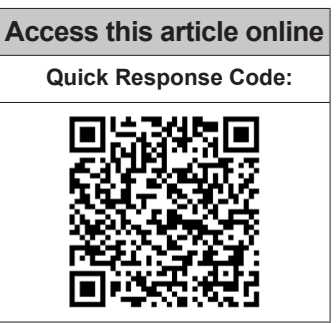

Website:

www.jlponline.org

DOI:

10.4103/JLP.JLP_141_18
Department of Pathology,

M.M. Institute of Medical Sciences and

Research, ${ }^{1}$ Department of Hematology, Maitri Diagnostic Lab, Ambala, Haryana, India

Address for correspondence:

Dr. Akshay Bali, Maitri Diagnostic Lab, First Floor Above Bali Clinic, Baldev Nagar,

Ambala - 134 003, Haryana, India. E-mail: aks23bali@gmail.

Submission: 20-10-2018 Accepted: 22-03-2019

\title{
M2G1G2 white blood cell flag by three-part automated hematology analyzer: A hint to dengue infection in appropriate clinical context
}

\author{
Maitrayee Roy, Akshay Bali ${ }^{1}$
}

\section{Abstract:}

BACKGROUND: Automated hematology analyzers often generate many flags which can provide important clues to the underlying hematological abnormality. Although pathologists are generally well versed in recognizing the importance of flags indicating potential leukemic blasts, their utility in hinting toward infectious etiology, especially during epidemics, is less well known. We analyzed any abnormal flags generated by a three-part automated hematology analyzer in serologically confirmed cases of dengue.

MATERIALS AND METHODS: The study included 28 patients diagnosed with serologically confirmed dengue infection. The venous samples were run on ABX Miros-60 three-part hematology analyzer. The complete blood count data and any abnormal flags were noted and correlated with peripheral blood film findings in all patients.

RESULTS: An abnormal white blood cell (WBC) flag was noted in all 28 patients, including two in whom all other hematological parameters were within normal limits. In 26 (93\%) patients, M2G1G2 WBC flag was noted while the samples of the remaining two patients generated M2G1 and L1M2G1G2 WBC flags, respectively.

CONCLUSION: An abnormal WBC flag, when correlated in appropriate clinical context, especially during a dengue outbreak, can aid in targeting the cohort of patients who will require immediate referral for serological confirmation.

Key words:

Dengue, M2G1G2 white blood cell flag, three-part hematology analyzer

\section{Introduction}

utomated hematology analyzer is Aubiquitous equipment in essentially all hematology laboratories having made time-consuming manual preliminary peripheral blood film (PBF) screening redundant. Electrical impedance principle, also dubbed as the Coulter principle, is utilized by almost every hematology analyzer to distinguish between red blood cells (RBCs), white blood cells (WBCs), and platelets. The three-part analyzers also utilize the same Coulter principle to

This is an open access journal, and articles are distributed under the terms of the Creative Commons Attribution-NonCommercial-ShareAlike 4.0 License, which allows others to remix, tweak, and build upon the work non-commercially, as long as appropriate credit is given and the new creations are licensed under the identical terms.

For reprints contact: reprints@medknow.com differentiate the WBCs into granulocytes, lymphocytes, and monocytes on the basis of cell size. The five-part analyzers works on flow cytometry light scatter principle for determining five-part leukocytes differential. ${ }^{[1]}$

In addition to the blood cell counts and indices, these analyzers often spew out many flags which more often remain as a footnote in the printout and are seldom paid attention to by the reporting pathologist. ${ }^{[2]}$ These flags, when analyzed in conjunction with blood cell counts and appropriate clinical context, may provide a potential clue to the underlying abnormality. Several

How to cite this article: Roy M, Bali A. M2G1G2 white blood cell flag by three-part automated hematology analyzer: A hint to dengue infection in appropriate clinical context. J Lab Physicians 2019;11:103-6. 
studies have observed good correlation between WBC flags and leukemic blasts in PBF. ${ }^{[3-8]}$ However, in a tropical nation where infectious diseases such as malaria and dengue pose a greater public health burden, the utility of the WBC flags in hinting toward an underlying infection is poorly understood and scarcely documented in the literature. ${ }^{[9-12]}$

We undertook this study to analyze any abnormal flags generated by a three-part automated hematology analyzer in serologically confirmed cases of dengue and correlate with other hematological parameters and PBF findings in these patients.

\section{Materials and Methods}

The study included 28 (16 females and 12 males) patients, aged 15-66 years, from semi-urban and rural areas who had presented with variable symptoms of high-grade fever, headache, muscle, and joint pain. All of them were diagnosed with serologically confirmed dengue fever.

During the initial presentation, venous blood samples were collected in K2 EDTA vials and run on ABX Micros-ES60 three-part hematology analyzer. The complete blood count ( $\mathrm{CBC}$ ) findings (including hemoglobin, hematocrit, RBC count, red cell indices, WBC count, and platelet count) and any WBC flags generated were noted. The PBFs, stained with Leishman stain, were examined in all the patients.

\section{Results}

An abnormal WBC flag was generated by the ABX micros-60 hematology analyzer in all 28 NS1 antigen-positive dengue patients. In 26 (93\%) patients, M2G1G2 WBC flag was noted while the samples of the remaining two patients generated M2G1 and L1M2G1G2 WBC flags, respectively [Figure 1].

Thrombocytopenia (platelet count $<150 \times 10^{9} / \mathrm{L}$ ) was present in $23(82 \%)$ patients, of which $8(28.6 \%)$ patients had platelet counts $<50 \times 10^{9} / \mathrm{L}$ at diagnosis. Leukopenia (WBC count $<4 \times 10^{9} / \mathrm{L}$ ) was detected in $10(35.7 \%)$ patients. The differential WBC counts highlighted relative lymphocytosis in 18 (64.3\%) patients. Hematocrit was increased in $4(14.3 \%)$ patients. On peripheral blood film examination, no blast was detected in any of the cases. Activated and plasmacytoid lymphocytes, defined as lymphocytes with intensely basophilic cytoplasm, moderate nucleocytoplasmic ratio, and eccentric nuclei, were observed in $8(28.6 \%)$ patients [Figure 2]. The important hematological parameters of all 28 patients are summarized in Table 1.

\section{Discussion}

Pathologists are generally well versed with the utility of WBC flags in suspecting acute leukemia which prompts us to quickly review the PBF for any atypical cells/blasts. Hoyer et al. analyzed WBC flags generated by Coulter STKS

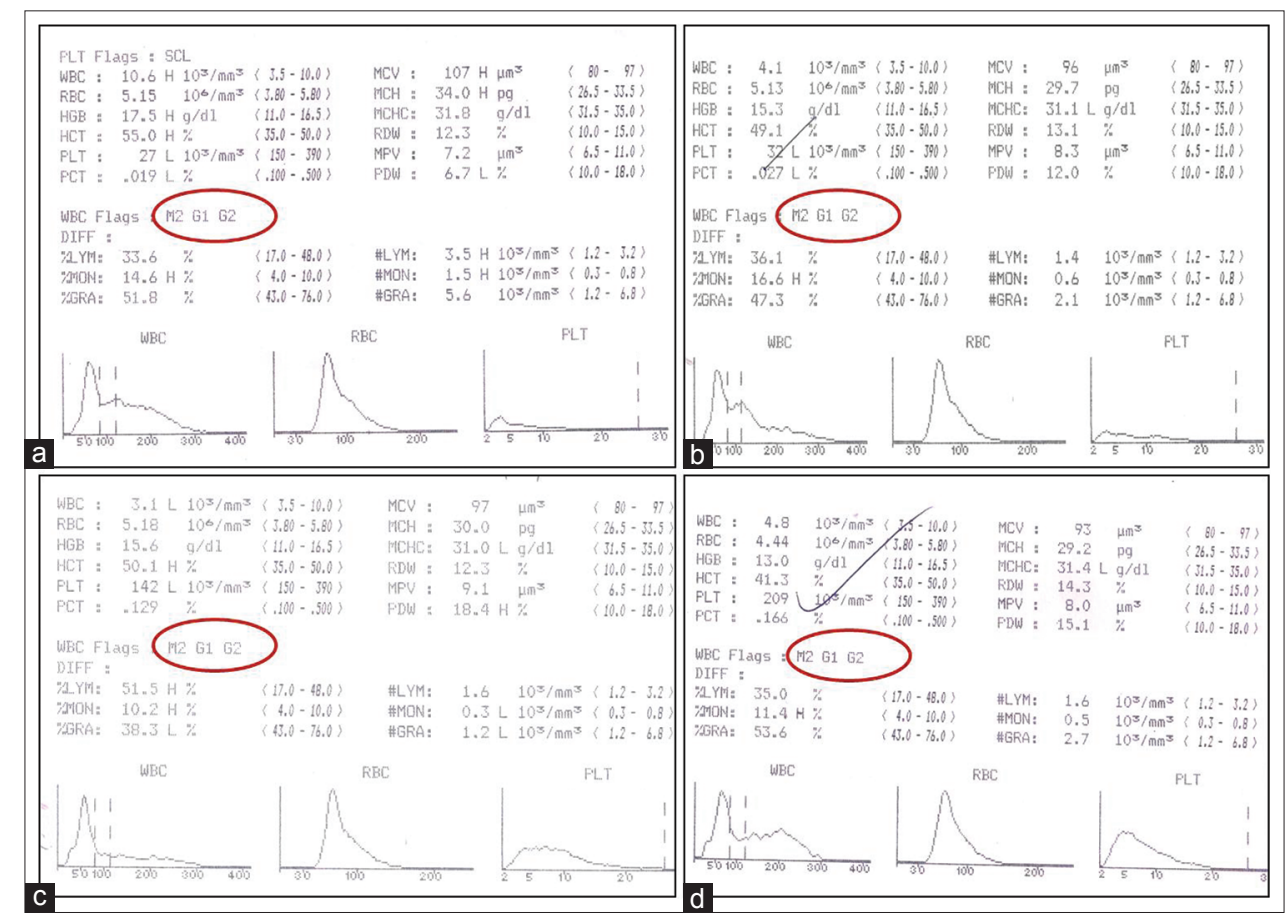

Figure 1: Complete blood count data from ABX Micros-60 three-part hematology analyzer: M2G1G2 WBC flag in dengue patients with (a) severe thrombocytopenia, raised hematocrit, and normal WBC count; (b) severe thrombocytopenia and borderline white blood cell count; (c) leukopenia and mild thrombocytopenia; (d) hematological parameters within normal range 


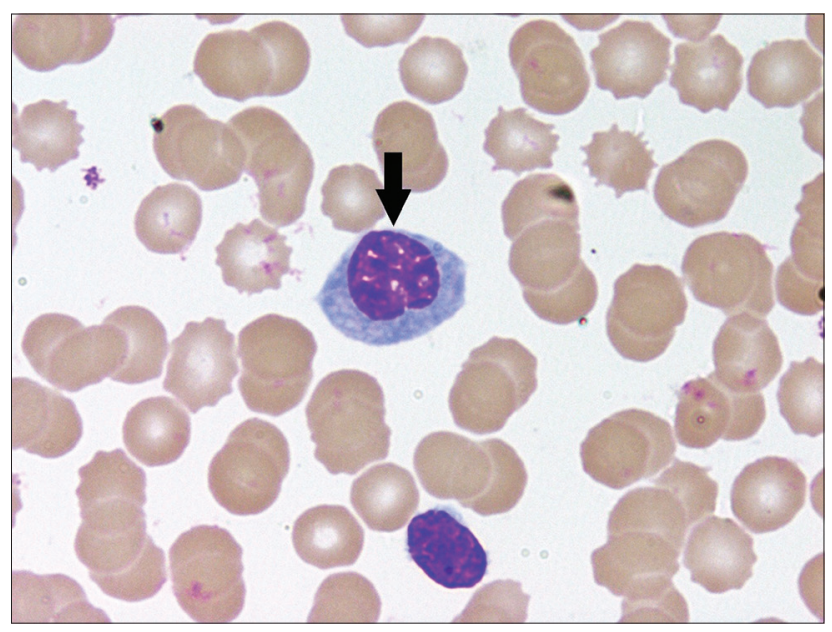

Figure 2: Peripheral blood film showing a plasmacytoid lymphocyte having intensely basophilic cytoplasm, moderate nucleocytoplasmic ratio, and eccentric nuclei (Leishman stain, $\times 1000$ )

Table 1: Hematological parameters in 28 serologically confirmed dengue patients

\begin{tabular}{lc}
\hline Parameters & Number of patients, $\boldsymbol{n}(\%)$ \\
\hline Platelet count $\left(\times 10^{9} / \mathrm{L}\right)$ & $8(28.6)$ \\
$<50$ & $15(53.6)$ \\
$50-<150$ & $5(17.8)$ \\
$\geq 150$ & \\
WBC count $\left(\times 10^{9} / \mathrm{L}\right)$ & $10(35.7)$ \\
$<4$ & $18(64.3)$ \\
$4-11$ & None \\
$>11$ & \\
Relative lymphocytosis & $18(64.3)$ \\
Present & $10(35.7)$ \\
Absent & \\
Activated/plasmacytoid lymphocytes & $8(28.6)$ \\
Present & $20(71.4)$ \\
Absent & \\
Hematocrit $(\%)$ & $4(14.3)$ \\
$>50$ & $24(85.7)$ \\
$\leq 50$ &
\end{tabular}

WBC: White blood cell

Hematology Analyzer in 110 patients of acute leukemia with circulating blasts. ${ }^{[4]}$ All samples with high WBC counts as well as most with normal/low WBC counts had elicited either definitive and/or suspect blast flags. ${ }^{[4]}$ Aulesa et al. noted over $90 \%$ diagnostic efficiency of immature granulocyte and blast flags in samples containing $>6 \%$ immature granulocytes and $>2 \%$ blasts, respectively. ${ }^{[5]}$ Kakkar and Kaur observed the WBC suspect blast flag M2 being generated by Advia-60 three-part hematology analyzer in all cases of acute leukemia accompanied by two nonblast flags, namely G1 and G2 ${ }^{[6]}$ Similar observation of M2G1G2 WBC flag in acute leukemia was reported in a study published by Kunal and Anne. ${ }^{[8]}$

However, in a resource-poor nation in tropical climate, where periodic outbreaks of malaria and dengue claim more lives annually, recognizing any consistent abnormal parameter and/or flags from the preliminary hematology analyzer data that can hint toward these underlying infection would be immensely beneficial in triggering early specific investigations. Yoon et al. observed high specificity of three parameters generated by a 5-part LH 780 hematology analyzer for identifying malaria in healthy and febrile individuals. These parameters included abnormal peak in WBC histogram before $35 \mathrm{fL}$, red dots in non-white cell zone and platelet-related flags suggesting platelet clumps or giant platelets. ${ }^{[9]}$ Sharma et al. also observed abnormalities in WBC scattergrams in patients with malaria. ${ }^{[12]}$

Dengue is a common arboviral infection with outbreaks and epidemics occurring almost every year in India from early September to November. One of the initial baseline investigations in a clinically suspected dengue infection is always a CBC. However, confirmation of dengue requires serological investigation by ELISA as mandated by government directives that may not always be readily available in all hospitals. In appropriate clinical scenario, recognizing important clue from $\mathrm{CBC}$ data can help in identifying the cohort of patients who will require immediate referral for serological confirmation. Thrombocytopenia is the most common hematological abnormality in dengue patients which can be accompanied by variable combination of leukopenia with relative lymphocytosis and raised hematocrit. Atypical and plasmacytoid lymphocytes are commonly detected on PBF examination. ${ }^{[13,14]}$ Oehadian et al. observed that flags such as increased atypical lymphocytes, high-fluorescent lymphocyte counts, and immature platelets were more commonly generated by Sysmex XE-5000 5-part hematology analyzer in dengue patients. ${ }^{[15]}$

We observed abnormal WBC flags generated by ABX Micros-60 three-part hematology analyzer in 28 serologically confirmed dengue patients, including two patients in whom all other hematological parameters were within normal range. The $\mathrm{CBC}$ data in all except two patients had M2G1G2 flag, while samples of remaining two patients had M2G1 and L1M2G1G2 flags, respectively, which are generally thought to hint at leukemic blasts. However, on thorough scrutiny of the PBFs, no blasts were detected and activated/plasmacytoid lymphocytes were observed in only $8(28.6 \%)$ patients.

\section{Conclusion}

With advancements in technology, the newer generation hematology analyzers are programmed to pick up a wide range of hematological abnormalities and have virtually replaced preliminary manual screening of 
slides in high-volume centers. However, in a community health center/hospital in the rural area with limited resources, during an epidemic, an abnormal WBC flag generated by a basic three-part hematology analyzer in appropriate clinical context can aid in early recognition of infectious etiology and referral for confirmatory serological investigations. On the upside, the absence of any abnormal hematological parameter and flag may reduce panic and overtesting during the dengue epidemic.

\section{Financial support and sponsorship}

Nil.

\section{Conflicts of interest}

There are no conflicts of interest.

\section{References}

1. Green R, Wachsmann-Hogiu S. Development, history, and future of automated cell counters. Clin Lab Med 2015;35:1-10.

2. Asad S, Ahmed I, Ali N. Utility of peripheral film findings and its correlation with automated analyzer - An audit from tertiary care hospital. J Lab Physicians 2017;9:1-4.

3. Kline A, Bird A, Adams L, Wale C, Edwards F, Perreira E. Identification of blast cells in the peripheral blood of patients with acute leukaemia using the technicon H-1. Clin Lab Haematol 1989;11:111-6.

4. Hoyer JD, Fisher CP, Soppa VM, Lantis KL, Hanson CA. Detection and classification of acute leukemia by the coulter STKS hematology analyzer. Am J Clin Pathol 1996;106:352-8.

5. Aulesa C, Pastor I, Naranjo D, Galimany R. Application of receiver operating characteristics curve (ROC) analysis when definitive and suspect morphologic flags appear in the new coulter LH 750 analyzer. Lab Hematol 2004;10:14-23.

6. Kakkar N, Kaur R. Utility of white blood cell suspect flags and histogram pattern in the detection of acute leukemia by advia-60 automated hematology analyzer. Indian J Pathol Microbiol 2004;47:322-6.

7. Guarner J, Atuan MA, Nix B, Mishak C, Vejajiva C, Curtis C, et al. Process to evaluate hematological parameters that reflex to manual differential cell counts in a pediatric institution. Clin Lab 2010;56:21-7.

8. Kunal O, Anne W. White blood cell suspect flags and white blood cell histogram pattern in acute leukemias. Panacea J Med Sci 2012;2:27-30.

9. Yoon J, Yun SG, Kim J, Jung YN, Koh YE, Nam JH, et al. Evaluation of the LH780 hematology analyzer for detection and therapeutic monitoring of malaria: Cross-reactivity with nucleated RBCs. Acta Trop 2016;164:425-30.

10. Sharma P, Bhargava M, Sukhachev D, Datta S, Wattal C. LH750 hematology analyzers to identify malaria and dengue and distinguish them from other febrile illnesses. Int J Lab Hematol 2014;36:45-55

11. Jain M, Gupta S, Jain J, Grover RK. Usefulness of automated cell counter in detection of malaria in a cancer set up -Our experience. Indian J Pathol Microbiol 2012;55:467-73.

12. Sharma S, Sethi N, Pujani M, Kushwaha S, Sehgal S. Abnormal WBC scattergram: A clue to the diagnosis of malaria. Hematology 2013;18:101-5.

13. Mehta RC, Goswami HM, Katara RK, Patel PS, Parikh UV, Vegad MM, et al. Importance of complete blood count and peripheral smear examination in early diagnosis of dengue patients. J Infect Dis Lett 2013;2:22-4.

14. Khatri K, Rajani A, Khalla AR. Plasmacytoid lymphocytes: A diagnostic clue to dengue infection. Int J Sci Res 2016;5:1002-5.

15. Oehadian A, Michels M, de Mast Q, Prihatni D, Puspita M, Hartantri $Y$, et al. New parameters available on sysmex XE-5000 hematology analyzers contribute to differentiating dengue from leptospirosis and enteric fever. Int J Lab Hematol 2015;37:861-8. 